\title{
A THIRD-ORDER NONLOCAL PROBLEM WITH NONLOCAL CONDITIONS
}

\section{LAZHAR BOUGOFFA}

Received 1 March 2003

\begin{abstract}
We study an equation with dominated lower-order terms and nonlocal conditions. Using the Riesz representation theorem and the Schauder fixed-point theorem, we prove the existence and uniqueness of a generalized solution.
\end{abstract}

2000 Mathematics Subject Classification: 35K35, 35B30, 35D05.

1. Introduction. Various problems arising in heat conduction, chemical engineering, plasma physics, thermoelasticity, and so forth, can be reduced to the nonlocal problems with integral conditions. This type of nonlocal boundary value problems has been investigated in $[1,2,3,4,5,6,8]$ for parabolic equations and in $[7,10]$ for hyperbolic equations. However, some partial differential equations of higher order with dominated low terms and nonlocal conditions are encountered when studying models for certain natural and physical processes. An example of such type of equations is the equation of longitudinal waves in a thin elastic stem taking into account the effects of transversal inertia [9]:

$$
\frac{\partial^{2} u}{\partial t^{2}}-\frac{\partial^{2} u}{\partial x^{2}}-\frac{\partial^{4} u}{\partial t^{2} \partial x^{2}}=0
$$

Another example is the equation of moisture transfer:

$$
\frac{\partial u}{\partial t}=\frac{\partial}{\partial x}\left(D \frac{\partial u}{\partial x}+A \frac{\partial^{2} u}{\partial x \partial t}\right)
$$

where $u$ is the concentration of moisture per unit, $D$ is the coefficient of diffusivity, and $A>0$ is the varying coefficient of Hallaire. Motivated by this, we study the equation

$$
l u=\frac{\partial^{3} u}{\partial t \partial x^{2}}-\frac{\partial u}{\partial t}+a(t, x) \frac{\partial^{2} u}{\partial x^{2}}+b(t, x) \frac{\partial u}{\partial x}+c(t, x) u=f(t, x)
$$

in the rectangular domain $\Omega=(0, T) \times(0,1)$.

To (1.3), we attach the nonlocal conditions

$$
\begin{gathered}
\int_{0}^{T} u(t, x) d t=0, \quad \forall x \in(0,1), \\
u(t, 0)=0, \quad \int_{0}^{1} u(t, x) d x=0, \quad \forall t \in(0, T) .
\end{gathered}
$$


We assume that the coefficients of $l$ are smooth and bounded on $\Omega$ :

$$
\begin{gathered}
0<a(t, x) \leq a_{0}, \quad 0<b(t, x) \leq b_{0} \frac{\sigma(x)}{\sqrt{2}}, \quad 0<c(t, x) \leq c_{0}, \\
\forall x \in(0,1), \quad \forall t \in(0, T), \quad \text { where } \sigma(x)=1-x .
\end{gathered}
$$

2. Generalized solution. Define the operator $l_{1}$ by

$$
l_{1} u=-\frac{\partial u}{\partial t}+a(t, x) \frac{\partial^{2} u}{\partial x^{2}}+b(t, x) \frac{\partial u}{\partial x}+c(t, x) u
$$

and

$$
F(t, x, u)=f(t, x)-l_{1} u .
$$

Then (1.3) can be assumed to have the form

$$
\frac{\partial^{3} u}{\partial t \partial x^{2}}=F(t, x, u)
$$

We introduce the function space

$$
\begin{gathered}
V=\left\{v: v \in L_{2}(\Omega), \frac{\sigma(x)}{\sqrt{2}} \frac{\partial v}{\partial x} \in L_{2}(\Omega), \frac{\partial v}{\partial t} \in L_{2}(\Omega), \frac{\partial^{2} v}{\partial x^{2}} \in L_{2}(\Omega),\right. \\
\left.\int_{0}^{T} v(t, x) d t=0, v(t, 0)=\int_{0}^{1} v(t, x) d x=0\right\} .
\end{gathered}
$$

The completion of this space, with respect to the norm

$$
\|v\|_{1,2, \sigma}^{2}=\int_{\Omega}\left[v^{2}+\frac{\sigma(x)^{2}}{2}\left(\frac{\partial v}{\partial x}\right)^{2}+\left(\frac{\partial v}{\partial t}\right)^{2}+\left(\frac{\partial^{2} v}{\partial x^{2}}\right)^{2}\right] d t d x
$$

is denoted by $\tilde{H}_{\sigma}^{1,2}(\Omega)$. Notice that $\tilde{H}_{\sigma}^{1,2}(\Omega)$ is a Hilbert space with

$$
(u, v)_{\tilde{H}_{\sigma}^{1,2}(\Omega)}=\int_{\Omega}\left[u v+\frac{\sigma^{2}(x)}{2} \frac{\partial u}{\partial x} \frac{\partial v}{\partial x}+\frac{\partial^{2} u}{\partial x^{2}} \frac{\partial^{2} v}{\partial x^{2}}\right] d t d x .
$$

For $v \in \tilde{H}_{\sigma}^{1,2}(\Omega)$, define the operator $M$ by

$$
\begin{aligned}
M v= & (1-x) \int_{0}^{t} \int_{0}^{x} v(\tau, \xi) d \xi d \tau-\int_{0}^{t} \frac{\partial^{2} v}{\partial x^{2}}(\tau, x) d \tau+\frac{(x-1)^{2}}{2} \int_{0}^{t} v(\tau, x) d \tau \\
& +\int_{0}^{x} J v(t, \xi) d \xi, \quad \text { where } J v=\int_{0}^{x} \frac{\partial v}{\partial t}(t, \xi) d \xi .
\end{aligned}
$$

DEFINITION 2.1. A function $u \in \widetilde{H}_{\sigma}^{1,2}(\Omega)$ is called a generalized solution to problem (1.3)-(1.4) if

$$
(u, v)_{\tilde{H}_{\sigma}^{1,2}(\Omega)}=(F(t, x, u), M v)_{L_{2}(\Omega)} \quad \text { for every } v \in \tilde{H}_{\sigma}^{1,2}(\Omega) .
$$


3. Existence and uniqueness theorem. In this section, we prove the existence and uniqueness of a generalized solution for the problem (1.3)-(1.4). For this, we first study the subsidiary problem

$$
l_{0} u \equiv \frac{\partial^{3} u}{\partial t \partial x^{2}}=F(t, x, 0)
$$

with integral conditions (1.4), where

$$
F(t, x, 0)=f(t, x)
$$

THEOREM 3.1. Let $F(t, x, 0) \in L_{2}(\Omega)$. Then there exists one and only one generalized solution $u_{0}$ of the subsidiary problem

$$
\begin{gathered}
l_{0} u \equiv \frac{\partial^{3} u}{\partial t \partial x^{2}}=F(t, x, 0), \\
\int_{0}^{T} u(t, x) d t=0, \quad \forall x \in(0,1), \\
u(t, 0)=0, \quad \int_{0}^{1} u(t, x) d x=0, \quad \forall t \in(0, T),
\end{gathered}
$$

such that

$$
c_{1}\left\|u_{0}\right\|_{1,2, \sigma} \leq\|F\|_{L_{2}(\Omega)},
$$

where $c_{1}$ is a positive constant.

Proof. For $F(t, x, 0) \in L_{2}(\Omega), \Psi(v)=(F, M v)_{L_{2}(\Omega)}$ is a bounded linear functional on $\tilde{H}_{\sigma}^{1,2}(\Omega)$.

Indeed,

$$
\left|(F, M v)_{L_{2}(\Omega)}\right| \leq\|F\|_{L_{2}(\Omega)}\|M v\|_{L_{2}(\Omega)} .
$$

By substituting the expression of $M v$ in (3.5) and using the Poincaré estimates

$$
\begin{gathered}
\int_{\Omega} v^{2}(t, x) d t d x \leq 4 \int_{\Omega}(1-x)^{2}\left(\frac{\partial v}{\partial x}\right)^{2} d t d x, \quad v(t, 0)=0 \\
\int_{\Omega}\left[\int_{0}^{t} v(\tau, x) d \tau\right]^{2} d t d x \leq 4 \int_{\Omega}(1-x)^{2} v^{2}(t, x) d t d x
\end{gathered}
$$

we find that $|\Psi(v)| \leq 4 \max \left\{2 T^{2}, 4\right\}\|F\|_{L_{2}(\Omega)}\|v\|_{1,2, \sigma}$.

Consider the scalar product $\left(l_{0}, M v\right)_{L_{2}(\Omega)}=\int_{\Omega} l_{0} u \cdot M v d t d x$; employing integration by parts and taking account of $v \in \tilde{H}_{\sigma}^{1,2}(\Omega)$, we obtain

$$
\left(\frac{\partial^{3} u}{\partial t \partial x^{2}}, M v\right)_{L_{2}(\Omega)}=(u, v)_{\tilde{H}_{\sigma}^{1,2}(\Omega)} .
$$

Thus, by the Riesz representation theorem, there exists a unique solution

$$
u_{0} \in \tilde{H}_{\sigma}^{1,2}(\Omega): \Psi(v)=(F, M v)_{L_{2}(\Omega)}=\left(u_{0}, v\right)_{\tilde{H}_{\sigma}^{1,2}(\Omega)}, \quad \forall v \in \tilde{H}_{\sigma}^{1,2}(\Omega) .
$$


Hence, $(u, v)_{\tilde{H}_{\sigma}^{1,2}(\Omega)}=\left(u_{0}, v\right)_{\tilde{H}_{\sigma}^{1,2}(\Omega)}$, that is, $u_{0}$ is a generalized solution. Letting $1 / c_{1}=$ $4 \max \left\{2 T^{2}, 4\right\}$, we obtain inequality (3.4).

LEMmA 3.2. The operator $l_{1}: \tilde{H}_{\sigma}^{1,2}(\Omega) \rightarrow L_{2}(\Omega)$ is bounded, that is, there exists a positive constant $c_{2}$ such that $\left\|l_{1} u\right\|_{L_{2}(\Omega)} \leq c_{2}\|u\|_{1,2, \sigma}$.

Proof. By using conditions (1.5), we directly obtain

$$
\left\|l_{1} u\right\|_{L_{2}(\Omega)}^{2} \leq 4\left(\left\|\frac{\partial u}{\partial t}\right\|_{L_{2}}^{2}+a_{0}^{2}\left\|\frac{\partial^{2} u}{\partial x^{2}}\right\|_{L_{2}}^{2}+b_{0}^{2}\left\|\frac{\partial u}{\partial x}\right\|_{L_{2, \sigma}}^{2}+c_{0}^{2}\|u\|_{L_{2}}^{2}\right),
$$

where $\|\partial u / \partial x\|_{L_{2, \sigma}}^{2}=\int_{\Omega}\left(\sigma^{2}(x) / 2\right)(\partial u / \partial x)^{2} d t d x$.

Hence, $\left\|l_{1} u\right\|_{L_{2}(\Omega)}^{2} \leq c_{2}^{2}\|u\|_{1,2, \sigma}^{2}$, where $c_{2}^{2}=4 \max \left\{1, a_{0}^{2}, b_{0}^{2}, c_{0}^{2}\right\}$.

Since $l_{1}$ is linear, then $l_{1}(\sqrt{2} \mu u)=\sqrt{2} \mu l_{1}(u)$ for an arbitrary $\mu$.

Let $l_{1, \mu}(w)=l_{1}(\sqrt{2} \mu w)$ for $\mu>1 / c_{1}$.

Now, consider the general case. The idea in the proof is to derive the results for the equation $l u=f$ with integral conditions (1.4).

THEOREM 3.3. Let $f(t, x) \in L_{2}(\Omega)$ and $|f(t, x)| \leq \lambda / \sqrt{2}$, where $\lambda$ is a constant. Then there exists at least one generalized solution $u_{0} \in \tilde{H}_{\sigma}^{1,2}(\Omega)$ to problem (1.3)-(1.4). Furthermore, the solution is uniquely determined if $c_{2}<c_{1}$.

Proof. Let $W=\left\{l_{1, \mu} w: l_{1, \mu} w \in L_{2}(\Omega),\left\|l_{1} u\right\|_{L_{2}(\Omega)}^{2} \leq \lambda^{2} T / \kappa^{2}\right\}$ be a closed ball, where $\kappa^{2}=c_{1}^{2}-1 / \mu^{2}$.

It is clear that

$$
|F(t, x, w)| \leq|f(t, x)|+\sqrt{\frac{c_{1}^{2}-k^{2}}{2}}\left|l_{1, \mu}(w)\right|,
$$

and we have $\|F(t, x, w)\|_{L_{2}(\Omega)}^{2} \leq c_{1}^{2} \lambda^{2} T / \kappa^{2}$ for all $l_{1, \mu} w \in W$.

From Theorem 3.1, there exists a unique generalized solution of the problem

$$
\frac{\partial^{3} u}{\partial t \partial x^{2}}=F(t, x, w)
$$

with integral conditions (1.4), so that

$$
(u, v)_{\widetilde{H}_{\sigma}^{1,2}(\Omega)}=(F, M v)_{L_{2}(\Omega)} .
$$

Define an operator $S: l_{1} w \in W \rightarrow u=S l_{1} w \in \tilde{H}_{\sigma}^{1,2}(\Omega), S(W) \subset W$.

Notice that $S$ is completely continuous. To show this, let $\left(l_{1} w\right)_{n},\left(l_{1} w\right)_{0} \in W$ and $\left\|\left(l_{1} w\right)_{n}-\left(l_{1} w\right)_{0}\right\|_{L_{2}(\Omega)}^{2} \rightarrow 0$, as $n \rightarrow \infty$.

Then, for $u_{n}=S\left(l_{1} w\right)_{n}, u_{0}=S\left(l_{1} w\right)_{0}$, we have

$$
\begin{aligned}
\left(u_{n}-u_{0}, v\right)_{\tilde{H}_{\sigma}^{1,2}(\Omega)} & =\left(F\left(t, x,(w)_{n}\right)-F\left(t, x,(w)_{0}\right), M v\right) \\
& =\left(\left(l_{1} w\right)_{n}-\left(l_{1} w\right)_{0}, M v\right)_{L_{2}(\Omega)} \text { for every } v \in \tilde{H}_{\sigma}^{1,2}(\Omega) .
\end{aligned}
$$


Now, from Theorem 3.1,

$$
c_{1}\left\|u_{n}-u_{0}\right\|_{1,2, \sigma} \leq\left\|\left(l_{1} w\right)_{n}-\left(l_{1} w\right)_{0}\right\|_{L_{2}(\Omega)} \longrightarrow 0 \quad \text { as } n \longrightarrow \infty .
$$

Again, taking a sequence $\left\{\left(l_{1} w\right)_{n}\right\} \subset W,\left\|\left(l_{1} w\right)_{n}\right\|_{L_{2}(\Omega)}^{2} \leq \lambda^{2} T / \kappa^{2}$. For $u_{n}=S\left(l_{1} w\right)_{n}$, we have $\left\|u_{n}\right\|_{L_{2}(\Omega)}^{2} \leq \lambda^{2} T / \kappa^{2}$, so a sequence $\left\{u_{n}\right\}$ is bounded in $\tilde{H}_{\sigma}^{1,2}(\Omega)$; therefore there exists a subsequence weakly convergent in $\tilde{H}_{\sigma}^{1,2}(\Omega)$.

Since any bounded set in $\tilde{H}_{\sigma}^{1,2}(\Omega)$ is compact in $L_{2}(\Omega)$, then there exists a subsequence, which we also denote by $\left\{u_{n}\right\}$, strongly convergent in $L_{2}(\Omega)$ to $u_{0}$, as $n \rightarrow \infty$. As $l_{1}$ is a bounded operator, $S$ is completely continuous, and so $S l_{1}$ is completely continuous. Thus, from Schauder's fixed-point theorem, there exists at least one fixed point $u_{0} \in W$ such that $u_{0}=S l_{1} u_{0}$ and $\left(u_{0}, v\right)_{\tilde{H}_{\sigma}^{1,2}(\Omega)}=\left(F\left(t, x, u_{0}\right), M v\right)_{L_{2}(\Omega)}$ for every $v \in \tilde{H}_{\sigma}^{1,2}(\Omega)$.

Now, assume that $u_{1}, u_{2}$ are distinct generalized solutions, then $\left(u_{1}-u_{2}, v\right)_{\tilde{H}_{\sigma}^{1,2}(\Omega)}=$ $\left(F\left(t, x, u_{1}\right)-F\left(t, x, u_{2}\right), M v\right)_{L_{2}(\Omega)}$ for all $v \in \tilde{H}_{\sigma}^{1,2}(\Omega)$.

From (3.4) and Lemma 3.2, we have

$$
\left\|u_{1}-u_{2}\right\|_{1,2, \sigma} \leq \frac{1}{c_{1}}\left\|l_{1} u_{1}-l_{1} u_{2}\right\| \leq \frac{c_{2}}{c_{1}}\left\|u_{1}-u_{2}\right\|_{1,2, \sigma}
$$

Thus, if $c_{2}<c_{1}$, then it gives a contradiction; therefore $u_{1}=u_{2}$.

\section{REFERENCES}

[1] G. W. Batten Jr., Second-order correct boundary conditions for the numerical solution of the mixed boundary problem for parabolic equations, Math. Comp. 17 (1963), 405-413.

[2] A. Bouziani and N.-E. Benouar, Mixed problem with integral conditions for a third order parabolic equation, Kobe J. Math. 15 (1998), no. 1, 47-58.

[3] B. Cahlon, D. M. Kulkarni, and P. Shi, Stepwise stability for the heat equation with a nonlocal constraint, SIAM J. Numer. Anal. 32 (1995), no. 2, 571-593.

[4] J. R. Cannon, The solution of the heat equation subject to the specification of energy, Quart. Appl. Math. 21 (1963), 155-160.

[5] Y. S. Choi and K.-Y. Chan, A parabolic equation with nonlocal boundary conditions arising from electrochemistry, Nonlinear Anal. 18 (1992), no. 4, 317-331.

[6] L. I. Kamynin, A boundary value problem in the theory of heat conduction with a nonclassical boundary condition, U.S.S.R. Comput. Math. and Math. Phys. 4 (1964), no. 6, 33-59.

[7] L. S. Pulkina, A non-local problem with integral conditions for hyperbolic equations, Electron. J. Differential Equations 1999 (1999), no. 45, 1-6.

[8] P. Shi, Weak solution to an evolution problem with a nonlocal constraint, SIAM J. Math. Anal. 24 (1993), no. 1, 46-58.

[9] M. Kh. Shkhanukov, A method for solving boundary value problems for third-order equations, Dokl. Akad. Nauk SSSR 265 (1982), no. 6, 1327-1330 (Russian).

[10] V. F. Volkodavov and V. E. Zhukov, Two problems for the string vibration equation with integral conditions and special matching conditions on the characteristic, Differ. Equ. 34 (1998), no. 4, 501-505.

Lazhar Bougoffa: Department of Mathematics, Faculty of Science, King Khalid University, P. O. Box 9004, Abha, Saudi Arabia

E-mail address: abogafah@kku.edu. sa 


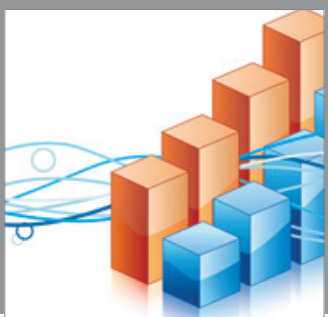

Advances in

Operations Research

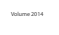

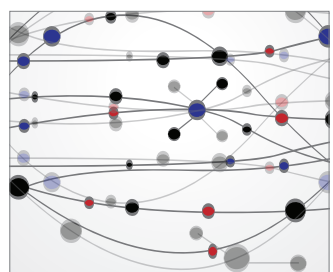

\section{The Scientific} World Journal
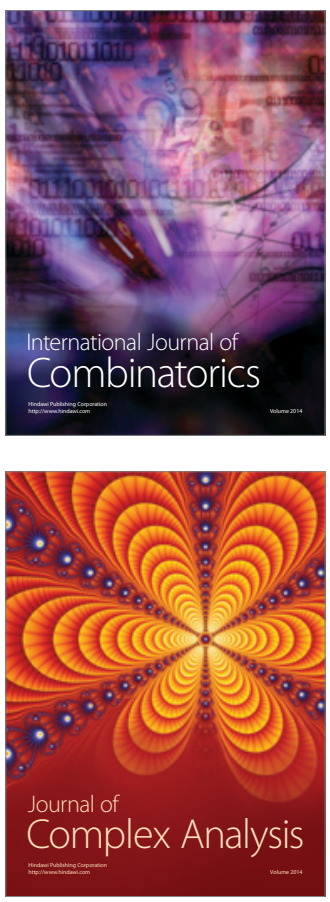

International Journal of

Mathematics and

Mathematical

Sciences
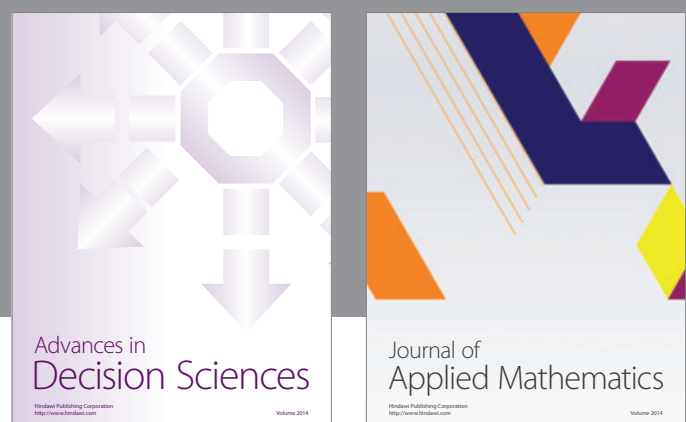

Journal of

Applied Mathematics
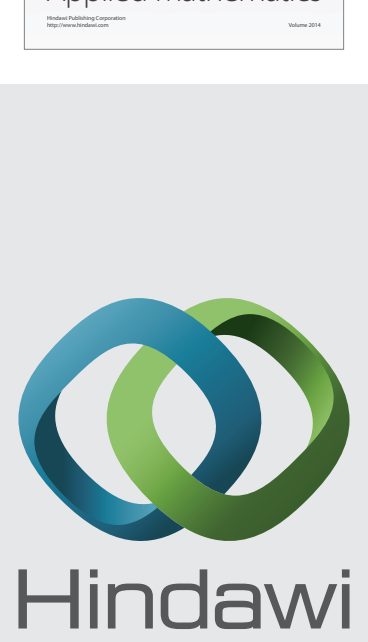

Submit your manuscripts at http://www.hindawi.com
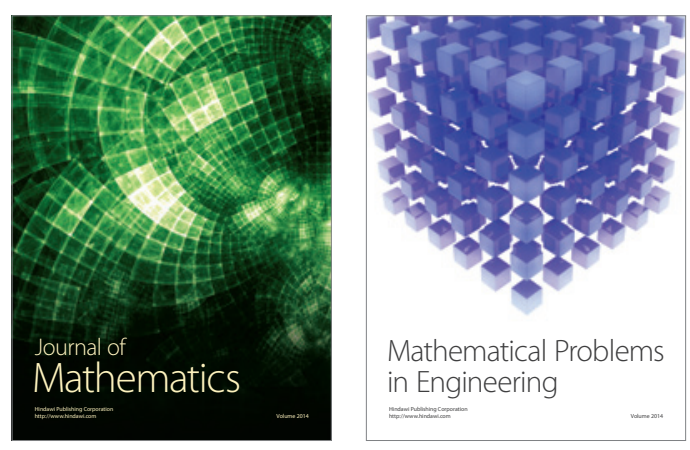

Mathematical Problems in Engineering
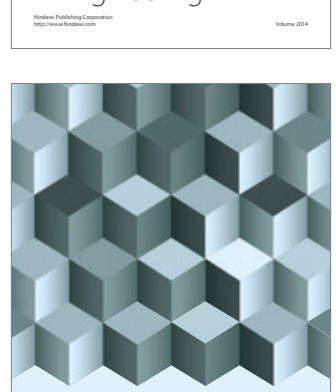

Journal of

Function Spaces
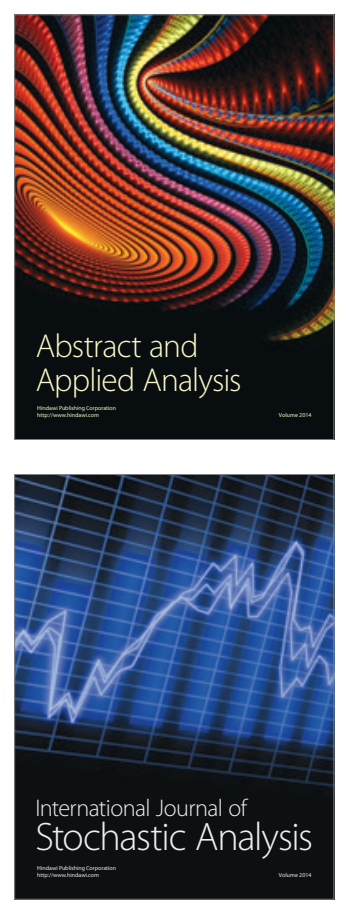

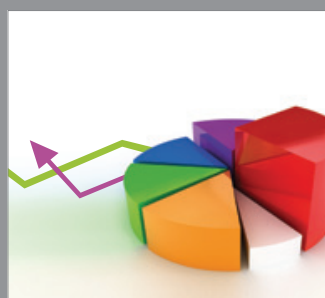

ournal of

Probability and Statistics

Promensencen
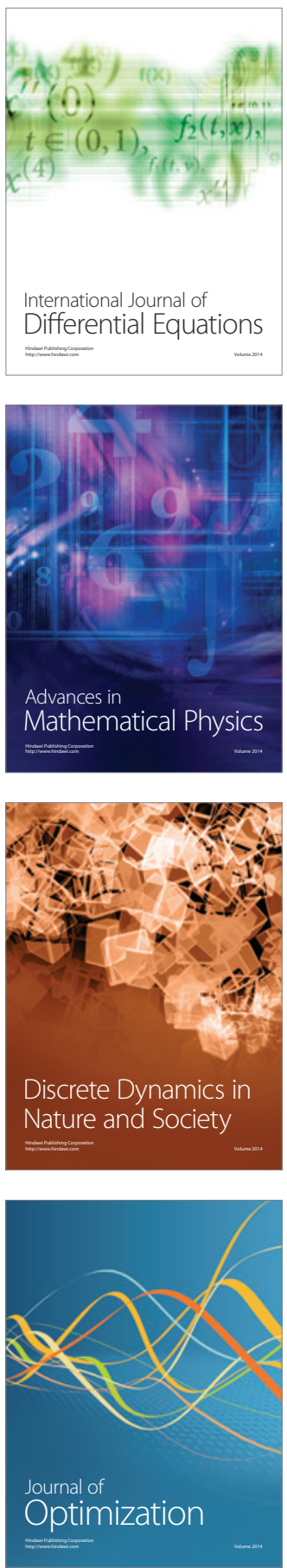